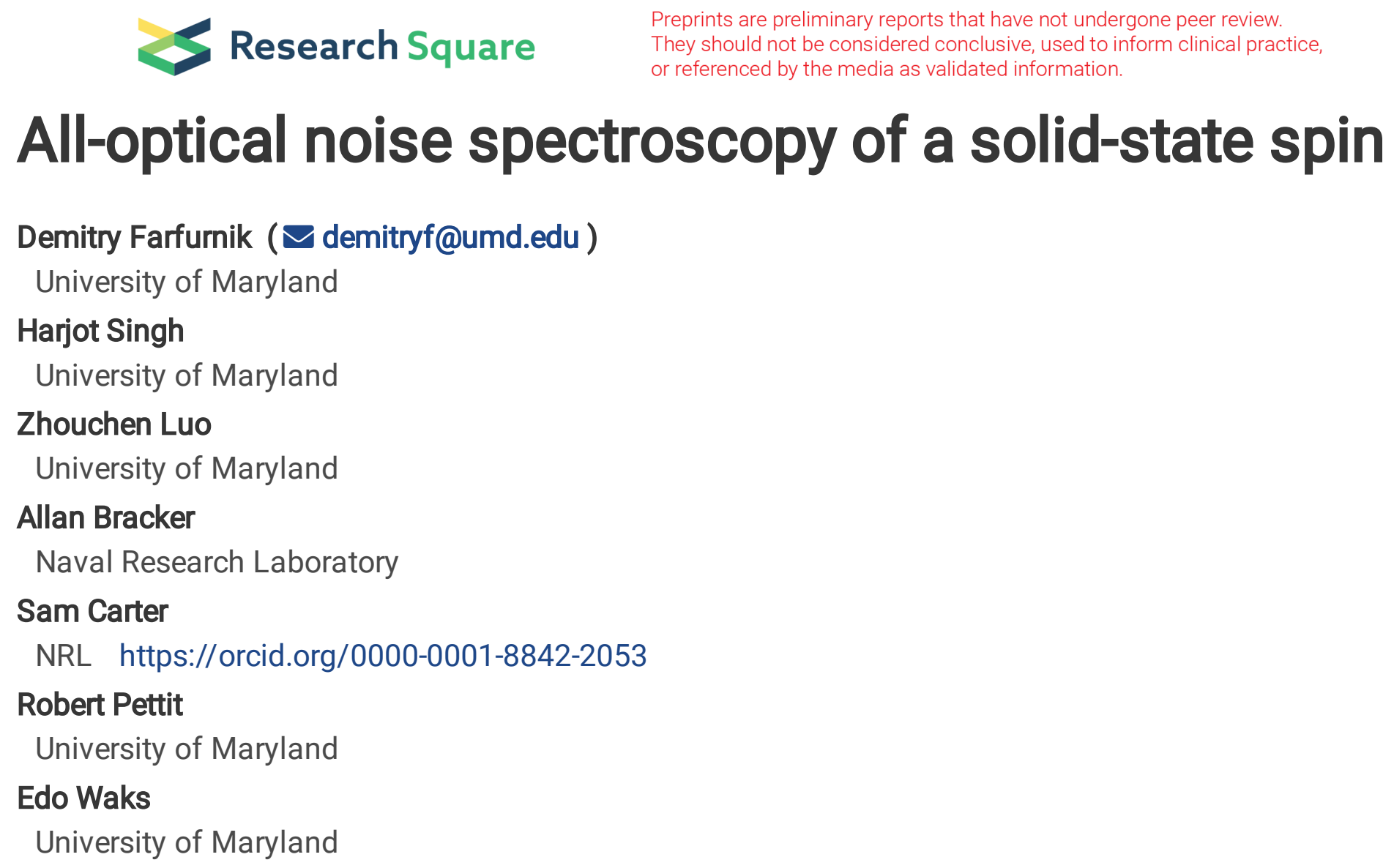

\title{
Letter
}

Keywords: all-optical noise spectroscopy, solid-state spin qubits, Raman rotations

Posted Date: November 30th, 2021

DOI: https://doi.org/10.21203/rs.3.rs-1068557/v1

License: (a) This work is licensed under a Creative Commons Attribution 4.0 International License. Read Full License 


\section{All-optical noise spectroscopy of a solid-state spin}

Demitry Farfurnik* ${ }^{1}$ Harjot Singh, ${ }^{1}$ Zhouchen Luo, ${ }^{1}$ Allan S.

Bracker, ${ }^{2}$ Samuel G. Carter, ${ }^{2}$ Robert M. Pettit, ${ }^{1,3}$ and E. Waks ${ }^{1}$

${ }^{1}$ Department of Electrical and Computer Engineering, Institute for Research in Electronics and Applied Physics, and Joint Quantum Institute, University of Maryland, College Park, MD, USA.

${ }^{2}$ Naval Research Laboratory, Washington, D.C., USA.

${ }^{3}$ Intelligence Community Postrdoctoral Research Fellowship Program, University of Maryland, College Park, MD, USA. 
Noise spectroscopy elucidates the fundamental noise sources in spin systems, which is essential for developing spin qubits with long coherence times for quantum information processing [1], communication [2], and sensing [3]. But noise spectroscopy typically relies on microwave coherent spin control to extract the noise spectrum [4-9], which becomes infeasible when there are highfrequency noise components stronger than the available microwave power. Here, we demonstrate an alternative all-optical approach to performing noise spectroscopy. Our approach utilises coherent Raman rotations [10, 11] of the spin state with controlled timing and phase to implement Carr-Purcell-MeiboomGill (CPMG) [12] pulse sequences. Analysing the spin dynamics under these sequences enables us to extract the noise spectrum of a dense ensemble of nuclear spins interacting with a single spin in a quantum dot, which has thus far only been modelled theoretically $[13,14]$. By providing large spectral bandwidths of over $100 \mathrm{MHz}$, our Raman-based approach could serve as an important tool to study spin dynamics and decoherence mechanisms for a broad range of solid-state spin qubits.

The coherent control of spin qubits can be used to conduct noise spectroscopy of their surrounding environment [4-9], in which the spin is used to probe the frequencies of the fluctuating fields generated by neighbouring nuclear and electronic spins as well as the strength of their interaction with the spin (i.e., the spectral density). Such noise spectroscopy using microwave fields has shed light on the fundamental noise processes of spin systems such as superconducting qubits $[4,5]$, nitrogen-vacancy centres in diamond [6,7], and gate-defined quantum dots $[8,9]$. However, the bandwidth of noise spectroscopy utilising microwave fields is limited by the rate of the spin rotation (i.e., the Rabi frequency), which must exceed the frequencies of the noise. As such, noise spectroscopy utilising microwave control becomes challenging in systems that feature noise spectra with frequencies greater than the power that can be achieved using microwave control.

An alternative approach for noise spectroscopy is to use optical fields. For example, optical measurements of resonance fluorescence [15] and Faraday rotation [16] have quantified the impact of slowly varying noise on the dynamics of single spins without applying coherent spin control. However, these approaches do not directly probe the coupling strengths of the fluctuating fields to the spin, which is essential information for the prediction of spin 
coherence times. In addition, the bandwidth of noise spectroscopy techniques that measure resonance fluorescence or Faraday rotation on a single spin has been orders of magnitude smaller than the high frequencies (up to $100 \mathrm{MHz}[13,14]$ ) associated with the noise sources that dictate spin dynamics and decoherence. Probing such high frequency noise of a single spin requires the application of sequences of all-optical coherent control pulses.

In this Letter, we demonstrate all-optical noise spectroscopy of a single solid-state spin utilising coherent control. Our approach utilises Raman rotation pulses with controllable timing, amplitude, and phase [10,11] to implement CPMG control sequences [12]. With this approach, we extract the noise spectrum of an ensemble of indium and arsenic nuclear spins (i.e., the Overhauser field) interacting with a single electron spin confined in an InAs/GaAs quantum dot, which has thus far only been modelled theoretically [13, 14]. Previously demonstrated microwave control of quantum dots has been orders of magnitude slower than required for noise spectroscopy due to the low g-factor $(\sim 0.4)[17]$ of the electron confined in the quantum dot. In contrast, the high spectral bandwidths of noise spectroscopy ( $>100$ $\mathrm{MHz}$ ) provided by our all-optical approach enable us to probe the Overhauser field, which features high frequencies due to the spread of the Larmor frequencies of the background nuclei at high magnetic fields (up to $2 \mathrm{~T}$ ). The extracted spectra verify a theoretical model that predicts two noise components (in-plane and out-of-plane to the external field) caused by an inhomogeneous strain field in the quantum dot environment [13, 14]. Understanding of such noise sources can quantify the achievable coherence times of spin qubits and predict the spin dynamics of such qubits for quantum information processing, communication, and sensing.

We perform the all-optical noise spectroscopy using a negatively charged quantum dot (see Methods) [18-25], which acts as a quantum probe for the fluctuations of nuclear spins in its environment. Under an external magnetic field applied perpendicular to the sample growth direction (Voigt Geometry), the electronic structure of these dots consists of an electron ground-state spin qubit $(\{|\uparrow\rangle,|\downarrow\rangle\})$ and two optically excited trion states $(\{\mid \uparrow \downarrow, \Uparrow$ \rangle$,|\uparrow \downarrow, \Downarrow\rangle\})[18]$. Spontaneous decay from the excited states leads to fluorescence emission of single photons with high efficiency and indistinguishability [20, 21, 23, 24]. The noise source (the Overhauser field of the indium and arsenic nuclear spins) that leads to the decoherence of the quantum dot spin consists of frequencies of $\sim$ tens of $\mathrm{MHz}[13,14]$ (the exact frequency depends on the magnitude of the external magnetic field). Here, we perform 


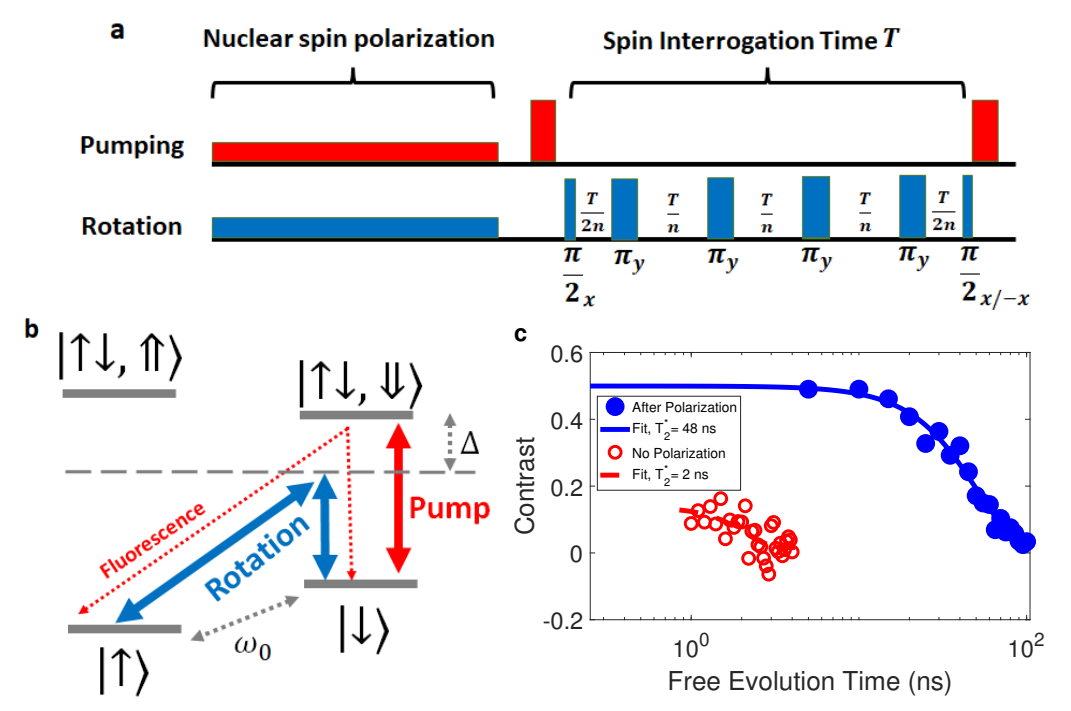

Fig. 1 | The realisation of the CPMG pulse sequences used for noise spectroscopy. a, Representative scheme of a CPMG sequence, with $n=4$ pulses and total interrogation time $t$, performed after 4 us of nuclear polarisation. The phase of the last pulse is alternated between $0^{\circ}$ and $180^{\circ}$ for normalisation purposes. The pumping and rotation amplitudes for nuclear polarisation (illustrated by the heights of the pulses) are weaker than those used for spin interrogation. b, The energy-level diagram of a negatively charged quantum dot under an external magnetic field in the Voigt geometry, including the optical pumping (solid red arrows) and Raman rotation (solid blue arrows) transitions used for noise spectroscopy, as well as the optical decay transitions from the excited state (dashed red arrows). Fluorescence collected from the $|\uparrow \downarrow, \Downarrow\rangle \rightarrow|\uparrow\rangle$ optical decay transition indicates the quantum dot spin state. c, The Ramsey experiment used to characterise our preliminary polarisation of the nuclear ensemble. The resulting spin dynamics without (dashed red line) and with (solid blue line) polarisation of the nuclear spin environment at an external field of $B=2.4 \mathrm{~T}$ shows the electron spin coherence time can be extended from $T_{2}^{*} \approx 2 \mathrm{~ns}$ (dashed red line) to $T_{2}^{*} \approx 48 \mathrm{~ns}$ (solid blue line).

noise spectroscopy of the Overhauser field utilising optical CPMG pulse sequences.

The CPMG sequences [12] implemented for noise spectroscopy are illustrated in Fig. 1a. After a preliminary 4- $\mathrm{s}$ s long stage of nuclear spin polarisation [11], we initialise the spin in the $\frac{|\uparrow\rangle+|\downarrow\rangle}{\sqrt{2}}$ state using an optical pumping pulse followed by a $\frac{\pi}{2}$-rotation pulse (see Methods). Then, we apply $n \geq 1$ equally spaced $\pi$-pulses that modify the temporal dynamics of the spin. After the application of a second $\frac{\pi}{2}$-pulse, a final optical pumping pulse induces a 
fluorescence signal that indicates the final spin state. Collecting the fluorescence while increasing the spin interrogation time $t$ (i.e., the time between the $\frac{\pi}{2}$-pulses), results in a decay of spin coherence represented by a coherence function, $C(t)$, which can be analysed for noise spectroscopy (see Methods).

To implement the all-optical coherent control pulses required for the CPMG sequences, we utilise detuned two-photon Raman excitations [10] (blue solid arrows in Fig. 1b) enabled by a modulated laser. We perform this modulation using an arbitrary waveform generator, which introduces spin rotation pulses with precise timing, phase, and Rabi frequencies of up to $\approx 150 \mathrm{MHz}$ (see Methods). To ensure that spin dephasing is minimal during the application of these pulses, we use the Raman coherent control to polarise the nuclear spin ensemble prior to the application of any CPMG sequence [11] (see Methods). As shown by a Ramsey experiment (Fig. 1c; see Methods), this 4-ps long nuclear polarisation step increases the inhomogeneous coherence time of the quantum dot spin from $T_{2}^{*} \approx 2$ ns (dashed red line in Fig. 1c) to $T_{2}^{*} \approx 48 \mathrm{~ns}$ (solid blue line in Fig. 1c), consistent with previous Ramsey experiments [11]. The spin dephasing time resulting from this coherent control is an order of magnitude longer than the spin rotation $\pi$-pulses, which allows us to use such pulses for the realisation of CPMG sequences.

We first apply the simplest form of the CPMG sequence, namely the Hahn-echo experiment consisting of a single $\pi$-pulse (Fig. 2a). Consistent with previous Hahn-echo measurements on quantum dots [14, 26, 27], the decay timescale of the spin dynamics (i.e., the coherence time) increases as a function of the external magnetic field, $\mathrm{B}$, up to $T_{2} \approx 1 \mu \mathrm{s}$ (solid blue line in Fig. 2a). This increase is associated with the Zeeman terms of the indium and arsenic nuclei dominating over the inhomogeneous broadening of these nuclei at high magnetic fields (Supplementary Note 1). Furthermore, the spin exhibits a two-stage decoherence behaviour that consists of a fast drop of the signal contrast, followed by a second decay (starting at $t \approx 100 \mathrm{~ns}$ ). This behaviour is analogous to the previously observed Hahn-echo spin dynamics of InGaAs quantum dots [14, 27]. Intuitively, the two stages of decoherence suggest that separate spectral components of the noise affect the spin dynamics at separate timescales. Simulation results (Fig. 2b), which consider such separate noise components associated with the strained nuclear environment of the quantum dot $[13,14]$ (Supplementary Note 1), agree with the experimental results, thereby confirming this hypothesis. To experimentally extract these spectral noise components, we apply CPMG sequences with 

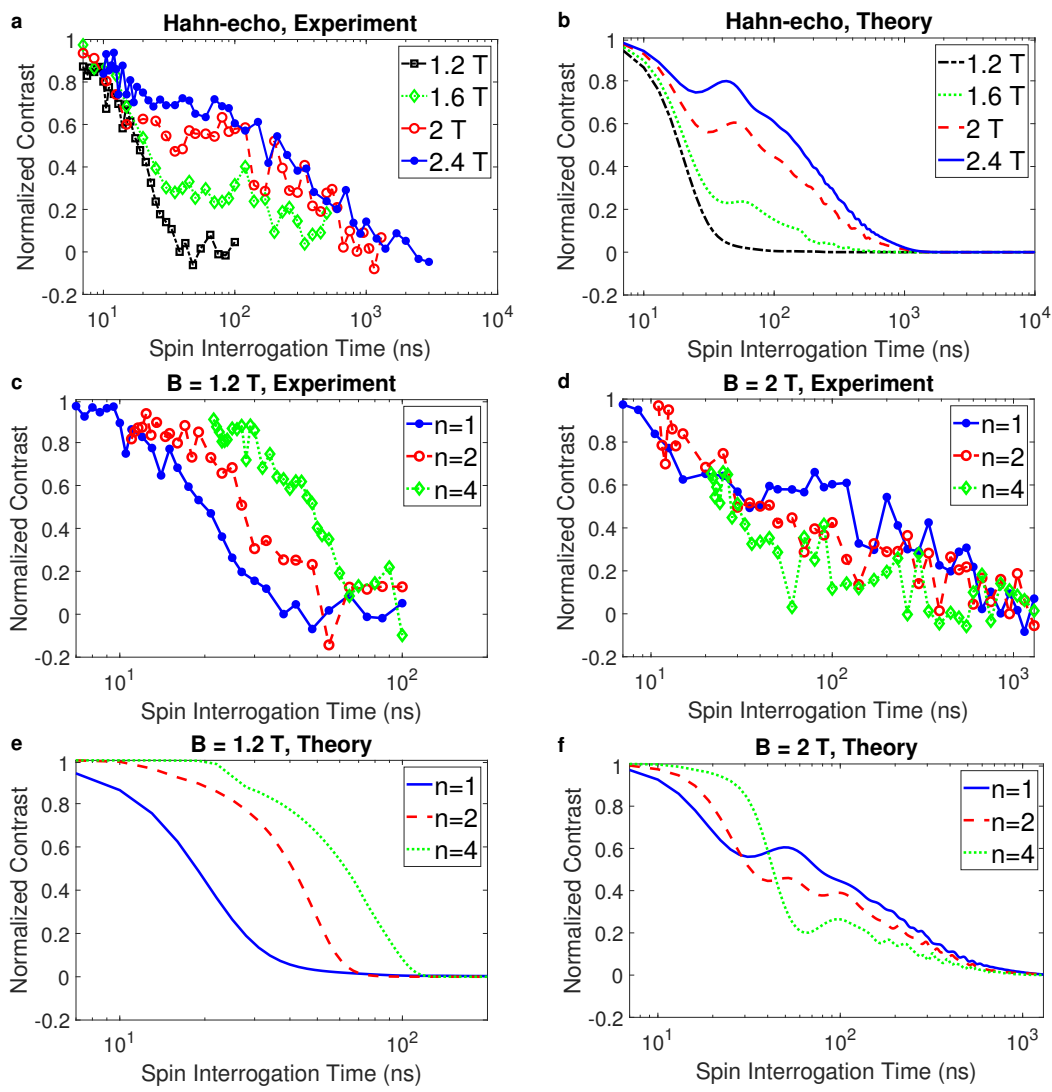

Fig. 2 | Coherence functions obtained from the application of CPMG sequences on a quantum dot spin. a-f, Normalised experimental and simulated coherence functions of a quantum dot spin under the application of CPMG sequences with $n$ pulses. The experimental results $(\mathbf{a})$ and simulations (b) of the Hahn-echo sequence $(n=1)$ at different magnetic fields, $B$. The experimental results $(\mathbf{c}, \mathbf{d})$ and simulations $(\mathbf{e}, \mathbf{f})$ of multi-pulse CPMG sequences at $B=1.2 \mathrm{~T}$ and $B=2 \mathrm{~T}$, respectively.

increasing numbers of pulses.

The temporal dynamics of the quantum dot spin under the application of such sequences is presented in Figs. 2c-f for different external magnetic fields between $1.2 \mathrm{~T}$ and $2 \mathrm{~T}$. As shown in Fig. 2c for $B=1.2 \mathrm{~T}$, the measured spin coherence times increase with $n$ as the decay timescale of the coherence function becomes longer. However, as shown in Fig. 2d, the two-stage spin decoherence profile for $B=2 \mathrm{~T}$ exhibits a more complicated behaviour as a function of $n$. Our simulations (shown in Fig. 2e and Fig. 2 f for $B=1.2 \mathrm{~T}$ and $B=2$ $\mathrm{T}$, respectively) considering two spectral components of the Overhauser field [13, 14] agree with the observed experimental behaviour. However, to understand these complex dynamics 
requires a comparison between the rate of application of these pulses with the frequencies of the noise spectra $[4,6-9,14,28-30]$.

We experimentally extract these noise frequencies by the numerical analysis of the measured coherence functions under the application of CPMG sequences with $n=1,2,4$ and 8 $\pi$-pulses (see Methods and Supplementary Note 2). The extracted spectral densities, plotted for external magnetic fields between $1.2 \mathrm{~T}$ and $2 \mathrm{~T}$ (data points in Fig. 3a-c), display a broad range of frequencies of up to $100 \mathrm{MHz}$. To characterise the behaviour of the noise as a function of the magnetic field, we fit the extracted spectra to Gaussian functions (solid blue lines in Fig. 3a-c). The central frequency of the noise (centre of the Gaussian fit) increases with the magnetic field up to $38 \mathrm{MHz}$ at $B=2 \mathrm{~T}$ (Fig. 3d), which is consistent with the increase of the nuclear Larmor frequencies. Meanwhile, the amplitude of the noise (at the central frequency) decreases with the magnetic field (Fig. 3e) as nuclear Zeeman interactions dominate over the broadening of the nuclei due to strain fields (Supplementary Note 1) $[13,14]$.

The extracted noise spectra verify a previously established theoretical model of the Overhauser field $[13,14]$ that allows us to understand the temporal dynamics of the quantum dot spin presented in Fig. 2. Using this model, we simulate the noise spectra that represent the hyperfine coupling of the quantum dot spin to indium and arsenic nuclear spins experiencing quadrupolar coupling to strain fields (solid red lines in Fig. 3a-c). The amplitudes of the simulated spectra consistently fit the experimentally extracted amplitudes (Fig. 3e) and indicate that the quantum dot spin interacts with 30,000 nuclei (Supplementary Note 1), in agreement with common predictions [14]. In addition, the theoretical model predicts two separate noise terms, perpendicular and parallel to the external magnetic field [13, 14]. The noise component perpendicular to the external field, $S_{\perp}(\omega)$, monotonically decreases with the frequency and qualitatively fits the experimentally extracted noise floor (i.e., the baselines of the Gaussian fits in Fig. 3a-c). This noise component dominates at low frequencies $(<10 \mathrm{MHz}$, Supplementary Note 1), thereby leading to the spin dynamics at long timescales (> $100 \mathrm{~ns}$ ) depicted in Fig. 2. Since the application rate of $\pi$-pulses in our CPMG sequences is faster than the low frequencies of $S_{\perp}(\omega)$, increasing the number of pulses slows down the decay of the spin dynamics at long timescales. For example, under an external field of $2 \mathrm{~T}$ (Fig. 2d), the decay of the spin dynamics at $t>100 \mathrm{~ns}$ is slower for $n=4$ (dotted green line) than for $n=1$ (solid blue line). In addition to the perpendicular noise 

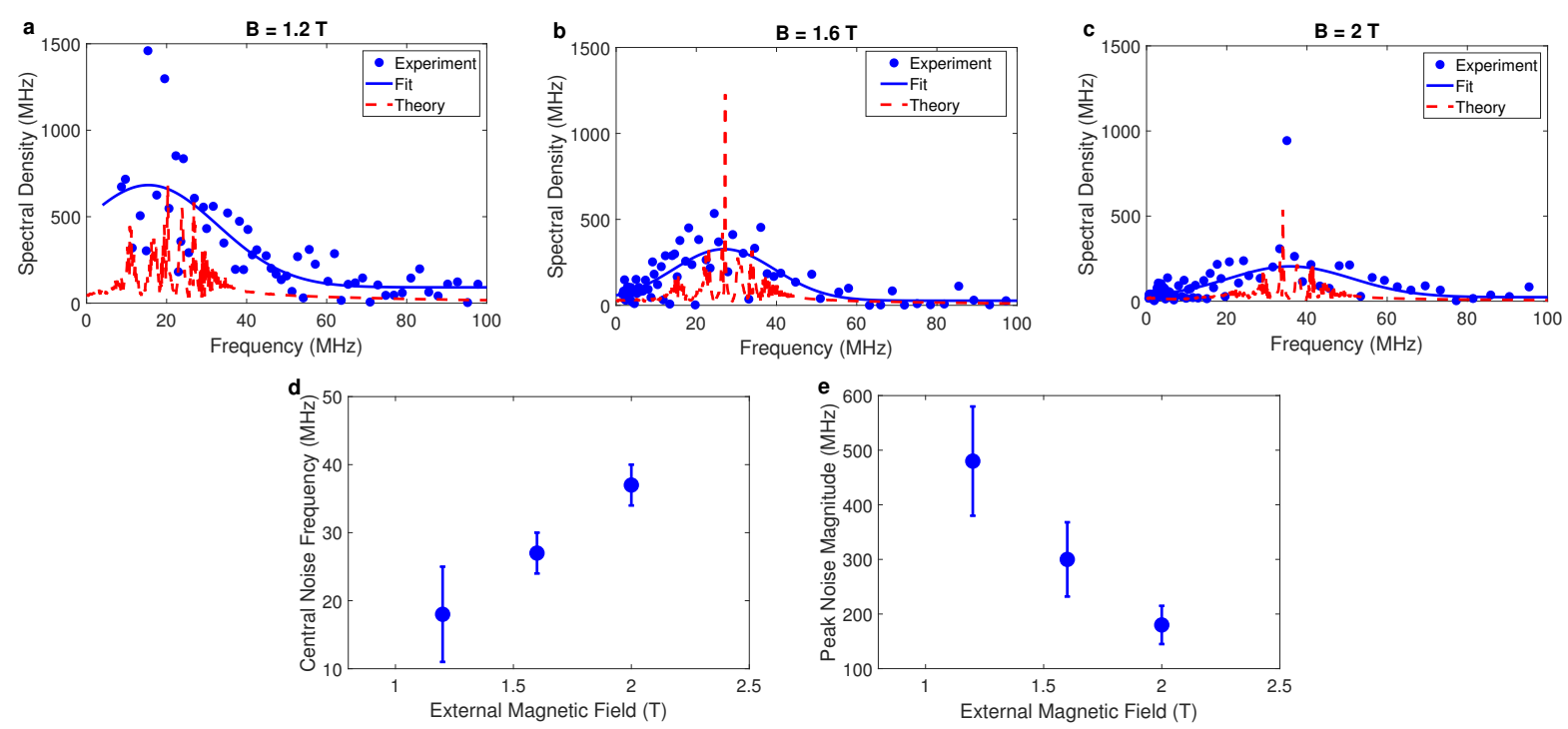

Fig. 3 Noise spectroscopy of the quantum dot environment. a-c, Noise spectroscopy of the nuclear spin ensemble interacting with a quantum dot, derived from spectral decomposition of CPMG experiments with $n=1,2,4$ and 8 pulses at varying magnetic fields of $B=1.2 \mathrm{~T}(\mathbf{a})$, $B=1.6 \mathrm{~T}(\mathbf{b})$, and $B=2 \mathrm{~T}(\mathbf{c})$. The blue dots represent experimental results, the solid blue lines represent the fitting of these results to Gaussian functions, and the dashed red lines represent simulations considering 30,000 nuclear spins. d, The central frequency and e, magnitude at the central frequency of the noise spectral density as a function of the magnetic field, extracted from the Gaussian fitting in a-c, with error bars obtained from a least-square fitting algorithm.

term, the theoretical model predicts high frequency noise, $S_{\|}(\omega)$, which arises in parallel to the direction of the external field $[13,14]$. This parallel term is stronger than the perpendicular term and consists of peaks corresponding to the nuclear Larmor frequencies broadened by the environmental strain field (Supplementary Note 1). The broad spectral features of $S_{\|}(\omega)$ lead to the observed contrast drop [30] at the short timescales depicted in Fig. 2 and quantify the ability of the CPMG sequences to extend the quantum dot spin coherence time. For example, the application rate of the $\pi$-pulses in our CPMG sequences is slower than the high frequency components of $S_{\|}(\omega)$ for $B=2 \mathrm{~T}$. As a result, the decay of the spin dynamics at timescales shorter than 100 ns does not improve by increasing the number of pulses (Fig. 2d). By analysing the obtained noise spectra, we learn that mitigating such high frequency noise to extend the quantum dot spin coherence time from the current state-of-the-art of a few microseconds $[14,26,27]$ to beyond $10 \mu$ s requires the application 
of dynamical decoupling sequences utilising hundreds of $\pi$-pulses.

However, here we were able to apply just eight $\pi$-pulses due to two mechanisms of spin relaxation. First, the natural spin relaxation of the quantum dot in our sample, $T_{1} \approx 1$ us, did not allow us to observe the expected spin dynamics under multi-pulse sequences beyond microsecond timescales. This relaxation time could be extended up to milliseconds by modifying the tunnel barrier of the sample [31]. Second, increasing the number of CPMG pulses in our measurements resulted in a dramatic contrast drop of the collected fluorescence as a function of $n$ [10] (Supplementary Note 3). This contrast drop is related to electron tunnelling due to the increase of the laser power associated with the addition of the pulses. Such electron tunnelling could be mitigated by enhancing the coupling of the quantum dot to the rotation laser by fabricating photonic structures on the sample, enabling spin rotations using lower laser power. These low power rotations could then enable multi-pulse sequences prolonging spin coherence times. Furthermore, the application of hundreds of pulses could enable the realisation of advanced pulse sequences for high resolution quantum sensing (e.g., "DYSCO" [7] sequences), and for the preservation of arbitrary spin states (e.g., XY8-based sequences [28]) for quantum information processing.

To conclude, we introduce an all-optical approach for noise spectroscopy and implement it to study the environment of InAs quantum dots, for which the application of microwave control is challenging. Leveraging the high Rabi frequencies and precise control capabilities of the Raman approach provides spectral bandwidths ( $>100 \mathrm{MHz}$ ) that enable the identification of high frequency noise spectra. Our method can be adopted to any solid-state system incorporating optically-active spin qubits. The extracted noise spectra can be used to predict spin dynamics under the application of various control sequences and to understand the behaviour of spin coherence, thereby evaluating the potential of spin qubits for the applications of quantum sensing, communication, and information processing.

[1] D. P. DiVincenzo, "The physical implementation of quantum computation," Fortschr. Phys. 48, 771-783 (2000).

[2] N. Gisin and R. Thew, "Quantum communication," Nat. Photonics. 1, 165-171 (2007).

[3] C. L. Degen, F. Reinhard, and P. Cappellaro, "Quantum sensing," Rev. Mod. Phys. 89, 
035002 (2017).

[4] J. Bylander et al., "Noise spectroscopy through dynamical decoupling with a superconducting flux qubit," Nat. Phys. 7, 565-570 (2011).

[5] Y. Sung et al., "Multi-level quantum noise spectroscopy," Nat. Commun. 12, 967 (2021).

[6] N Bar-Gill et al., "Suppression of spin-bath dynamics for improved coherence of multi-spinqubit systems," Nat. Commun. 3, 858 (2012).

[7] Y. Romach et al., "Measuring Environmental Quantum Noise Exhibiting a Nonmonotonic Spectral Shape," Phys. Rev. Applied 11, 014064 (2019).

[8] F. K. Malinowski et al., "Notch filtering the nuclear environment of a spin qubit," Nat. Nanotechnol. 12, 16-20 (2017).

[9] K. W. Chan et al., "Assessment of a silicon quantum dot spin qubit environment via noise spectroscopy," Phys. Rev. Applied 10, 044017 (2018).

[10] J. H. Bodey et al., npj Quantum Inf. 5, 95 (2019).

[11] D. A. Gangloff et al., "Quantum interface of an electron and a nuclear ensemble," Science 364, 62-66 (2019).

[12] S. Meiboom and D. Gill, "Modified spin-echo method for measuring nuclear relaxation times," Rev. Sci. Instrum. 29, 688-691 (1958).

[13] C. Bulutay, "Quadrupolar spectra of nuclear spins in strained inxga1-xas quantum dots," Phys, Rev. B 85, 115313 (2012).

[14] R. Stockill et al., Nat. Commun. 7, 12745 (2016).

[15] A. V. Kuhlmann et al., "Charge noise and spin noise in a semiconductor quantum device," Nat. Phys. 9, 570-575 (2013).

[16] R. Dahbashi, J. Hubner, F. Berski, K. Pierz, and M. Oestreich, "Optical spin noise of a single hole spin localized in an (inga)as quantum dot," Phys. Rev. Lett. 112, 156601 (2014).

[17] M. Kroner et al., "Optical detection of single-electron spin resonance in a quantum dot," Phys. Rev. Lett. 100, 156803 (2008).

[18] M. Bayer et al., "Fine structure of nuclear and charged excitons in self-assembled in(ga)as/(al)gaas quantum dots," Phys. Rev. B 65, 195315 (2002).

[19] W. B. Gao, P. Fallahi, E. Togan, J. Miguel-Sanchez, and A. Imamoglu, "Observation of entanglement between a quantum dot spin and a single photon," Nature 491, 426-430 (2012).

[20] X. Ding et al., "On-demand single photons with high extraction efficiency and near-unity 
indistinguishability from a resonantly driven quantum dot in a micropillar," Phys. Rev. Lett. 116, $020401(2016)$.

[21] N. Somaschi et al., "Near-optimal single-photon sources in the solid state," Nat. Photonics 10, $340(2016)$.

[22] P. Lodahl, "Quantum-dot based photonic quantum networks," Quantum. Sci. Technol. 3, 013001 (2018).

[23] E. Schöll et al., "Resonance fluorescence of gaas quantum dots with near-unity photon indistinguishability," Nano Lett. 19, 2404-2410 (2019).

[24] N. Tomm et al., "A bright and fast source of coherence single photons," Nat. Nanotechnol. 16, 399-403 (2021).

[25] M. H. Appel et al., "Coherent spin-photon interface with waveguide induced cycling transitions," Phys. Rev. Lett. 126, 013602 (2021).

[26] D. Press et al., "Ultrafast optical spin echo in a single quantum dot," Nat. Photonics. 4, 367-370 (2010).

[27] A. Bechtold et al., "Three-stage decoherence dynamics of an electron spin qubit in an optically active quantum dot," Nat. Phys. 11, 1005-1008 (2015).

[28] D. Farfurnik et al., "Optimizing a dynamical decoupling protocol for solid-state electronic spin ensembles in diamond," Phys. Rev. B 92, 060301(R) (2015).

[29] L. Cywinski, R. M. Lutchyn, C. P. Nave, and S. Das Sarma, "How to enhance dephasing time in superconducting qubits," Phys. Rev. B 77, 174509 (2008).

[30] Rogerio de Sousa, "Electron spin as a spectrometer of nuclear-spin noise and other fluctuations," in Electron Spin Resonance and Related Phenomena in Low-Dimensional Structures, Topics in Applied Physics, Vol. 115 (Springer, Berlin, 2009) pp. 183-220.

[31] G. Gillard et al., "Fundamental limits of electron and nuclear spin qubit lifetimes in an isolated self-assembled quantum dot," npj Quantum Inf. 7, 43 (2021).

\section{METHODS}

Sample Growth. We used a sample grown by molecular beam epitaxy consisting of InAs quantum dots embedded in a GaAs p-i-n-i-n diode. The diode is an optical cavity, sandwiched between distributed Bragg reflectors, consisting of 10 and 4 periods of GaAs/AlAs 
layers below and above the cavity, respectively. The diode enabled the charging of the quantum dot with a single electron by the application of a voltage bias. The cavity directed the fluorescence emitted from the dot toward the collection port, thereby enhancing the measured signals.

Experimental Setup. The sample was mounted in a low vibration He exchange gas cryostation with a base temperature of $\sim 4.3 \mathrm{~K}$ (attocube attoDRY1000). A superconducting coil provided magnetic fields of 1-3 $\mathrm{T}$ perpendicular to the growth direction of the quantum dot (Voigt geometry), under which we performed noise spectroscopy of the quantum dot environment. Note, we observed agreement between the theoretical and experimental data (Figs. 2-3) considering magnetic fields that were 1.25 times weaker than those that were set on the magnet controller. We attribute this factor to attenuations in the cryostation and misalignment of the sample from the main axis of the magnet.

Two circularly polarised $\sim 920 \mathrm{~nm}$ continuous wave laser (MSquared SOLSTIS and New Focus Velocity TLB-6700) beams modulated through electro-optical modulators (EOSPACE AZ-0S5-10-PFA-PFA-930 and iXblue NIR-MX950-LN-20-P-P-FA-FA) introduced the optical pumping and spin rotation necessary for the all-optical control. Mounting the modulators on a temperature-stabilised copper plate and periodically calibrating their DC bias input provided an on/off output power extinction ratio of over three orders of magnitude. This extinction ratio ensured that optical pumping and spin rotation were negligible when the voltage input of the modulator was set to zero. Before and after the application of any rotation pulse sequence (Ramsey or CPMG), we optically pumped the $|\downarrow\rangle \leftrightarrow|\uparrow \downarrow, \downarrow\rangle$ transition and collected fluorescence emitted via the $|\uparrow \downarrow, \Downarrow\rangle \rightarrow|\uparrow\rangle$ transition. While such optical pumping initialised the spin in the $|\uparrow\rangle$ state, the emitted fluorescence signal indicated the spin state prior to the pumping pulse (as this fluorescence is only associated with the probability of the $|\downarrow\rangle$ state).

The fluorescence signal was collected from an objective lens with a numerical aperture of 0.68 , spectrally filtered through a $0.07 \mathrm{~nm}$ full width half maximum Gaussian bandpass filter (WL Photonics WLTF-NE-P-930) and detected on a superconducting nanowire single photon detector (Opus one $950 \mathrm{~nm}$ ). To optimise the collection signal-to-noise ratio, motorised quarter and half waveplates rejected the circularly polarised laser light directly reflected from the sample by six orders of magnitude. Finally, three microwave waveforms introduced by a $65 \mathrm{Gs} / \mathrm{S}$ arbitrary waveform generator (Keysight M8195A) were used to 
synchronise between the experimental equipment as follows. First, pulses of a harmonic signal with frequency $\frac{\omega_{0}}{2}$ were injected to one electro-optical modulator to introduce spin rotations. Then, a waveform consisting of square pulses was injected to the second modulator to switch the pumping laser on and off. A final square wave was then used to trigger the time-resolved detector.

Realisation of Pulse Sequences. To realise the Ramsey sequence, we first prepared the quantum dot spin in the $\frac{|\uparrow\rangle+|\downarrow\rangle}{\sqrt{2}}$ state by resonantly pumping the $|\downarrow\rangle \leftrightarrow|\uparrow \downarrow, \Downarrow\rangle$ transition to initialise the spin in the $|\uparrow\rangle$ state, followed by a rotation $\frac{\pi}{2}$-pulse. Then, the spin freely evolved for a given time. Finally, an additional $\frac{\pi}{2}$-pulse projected the spin state back to either of the ground states $|\uparrow\rangle,|\downarrow\rangle$ (depending on the phase of the $\frac{\pi}{2}$-pulse) and the spin coherence was measured using an optical pumping pulse. Collecting fluorescence while varying the free evolution time resulted in the Ramsey decay function. Separate results involving final projections to the $|\uparrow\rangle$ and $|\downarrow\rangle$ states were normalised with respect to each other to cancel technical drifts. The result was normalised to provide a contrast value of 1 for time $t=0$ (Fig. 1c).

The realisation of CPMG sequences (Fig. 1a) was similar to that of the Ramsey sequence, with the additional application of $n \pi$-pulses between the first and second $\frac{\pi}{2}$-pulses. The phase of the $\pi$-pulses was shifted by $90^{\circ}$ from the phase of the $\frac{\pi}{2}$-pulses to mitigate potential contrast drop due to fluctuations in the amplitude and phase of the rotation field [12, 28]. The total spin interrogation time was varied to extract the coherence functions depicted in Fig. 2.

Decomposition of the noise spectrum. To extract the noise spectrum of the Overhauser field from the results of the CPMG experiments, we treated the field as a semi-classical noise source $[6,14]$ with a spectral density of $S(\omega)$. In this treatment, the spin dynamics under the application of a pulse sequence satisfy $[29,30]$

$$
\chi(t)=\int_{0}^{\infty} \frac{S(\omega) F(\omega t)}{\pi \omega^{2}} d \omega,
$$

where $F(\omega)$ is the filter function that represents a control sequence in the frequency domain, and $\chi(t)=-\ln [C(t)]$. We used the measured coherence functions, $C(t)$, and known CPMG filter functions [29] for $n=1,2,4$ and 8 , to spectrally deconvolve equation (1) and extract the noise spectra [7] (see full algorithm in Supplementary Note 2).

Optimisation of Experimental Parameters. The Rabi frequency of spin rotation 
utilizing Raman control is given by $[10,11,14,26] \Omega_{R}=\frac{\Omega^{2}}{\Delta}$, where $\Omega^{2}$ is linearly proportional to the laser power, $P$, and $\Delta$ is the spectral detuning of the laser from the optical transitions of the quantum dot. Considering the adiabatic elimination principle, $\Delta \gg \Omega$ must be satisfied to avoid the population of the excited states $[10,11,14,26]$. Maximizing the Rabi frequency $\Omega_{R}$ by increasing the laser power or reducing the detuning would reduce the duration of the $\pi$-pulses and provide access to spin dynamics at short timescales, thereby increasing the bandwidth of the noise spectroscopy (Supplementary Note 2). However, the detuning cannot be reduced arbitrarily due to potential population of the excited states, and the laser power cannot be increased arbitrarily due to laser-induced tunnelling that leads to spin relaxation (Supplementary Note 3). As a result, we optimised the parameters for the rotation laser and found $\Delta \approx 535 \mathrm{GHz}(1.5 \mathrm{~nm})$ and $P \approx 15 \mu \mathrm{W}$ for the observation of eight Rabi oscillations at a frequency of $\Omega_{R} \approx 150 \mathrm{MHz}$.

Finally, optimised polarisation of the nuclear ensemble requires simultaneous optical pumping and spin rotation at rates on the order of the Larmor components of the nuclear spins [11]. For a magnetic field of $B=2.4 \mathrm{~T}$, for example, a rotation laser power of $2 \mu \mathrm{W}$ and optical pumping power of $5 \mathrm{nW}$ provided the optimal coherence time result of $T_{2}^{*} \approx 48$ ns, presented in Fig. 1c. Considering a $\Gamma_{0} \approx 250 \mathrm{MHz}$ linewidth of the quantum dot excited states, these optical powers translate to a rotation rate of $\approx 20 \mathrm{MHz}$ and a pumping rate of $\approx 27.5 \mathrm{MHz}$, consistent with previous work [11].

\section{ACKNOWLEDGEMENTS}

We thank M. Atatüre, C. Le Gall, D. A. Gangloff, N. Bar-Gill, Y. Romach, and D. S. Smirnov for fruitful discussions. This work has been supported by the Physics Frontier Center at the Joint Quantum Institute, the National Science Foundation (Grants PHY1415485 and ECCS1508897), and the ARL Center for Distributed Quantum Information (Grant W911NF1520067). D. F. acknowledges support by the Fulbright Postdoctoral Fellowship and the Israel Council for Higher Education Quantum Science and Technology Scholarship. A.S.B. and S.G.C acknowledge support from the U.S Office of Naval Research. R.M.P. acknowledges support through an appointment to the Intelligence Community Postdoctoral Research Fellowship Program at the University of Maryland, administered by Oak Ridge 
Institute for Science and Education through an interagency agreement between the U.S. Department of Energy and the Office of the Director of National Intelligence.

\section{AUTHOR CONTRIBUTIONS}

D.F. and E.W. conceived and designed the experiment. D.F. and H.S. performed measurements. D.F. and Z.L. constructed the experimental setup. D.F. analysed experimental results, performed simulations, and wrote the manuscript. A.S.B. and S.G.C. provided the sample grown by molecular beam epitaxy. All authors contributed to scientific discussions and the preparation of the manuscript.

\section{COMPETING INTERESTS}

The authors declare no competing interests. 


\section{Supplementary Files}

This is a list of supplementary files associated with this preprint. Click to download.

- 20211109NoiseSpectroscopySupp.pdf 